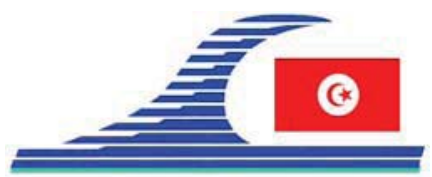

Conférence Méditerranéenne Côtière et Maritime EDITION 1, HAMMAMET, TUNISIE (2009)

Coastal and Maritime Mediterranean Conference

Disponible en ligne - http://www.paralia.fr - Available online

\title{
Hydrodynamique du golfe de Gabès déduite à partir des observations de courants et de niveaux
}

\section{Mohamed Jamel HATTOUR ${ }^{1}$, Chérif SAMMARI ${ }^{2}$, Sassi BEN NASSRALLAH ${ }^{3}$}

1. Ecole Nationale d'Ingénieurs de Monastir, Rue Ibn Eljazar, 5019 Monastir, Tunisie. hattour@yahoo.fr

2. Institut National des Sciences et Technologies de la Mer 28 rue du 2 mars 1934, 2025 Salammbô, Tunisie.

3. Laboratoire d'Etudes des Systèmes Thermiques et Energétiques, Rue Ibn Eljazar, 5019 Monastir, Tunisie.

\section{Résumé :}

L'objectif de cette étude est de vérifier et d'expliquer la dynamique dans le littoral du golfe de Gabès (Côtes Est de Tunisie) par des observations in situ. Nous avons effectué différentes mesures de hauteur d'eau et de courant en quelques points significatifs de cette région qui, avec l'Adriatique, bénéficient de la plus grande amplitude de marée en mer Méditerranée.

Cette étude présente les premiers résultats des analyses des séries temporelles.

Les instruments utilisés sont des ADCP (Accoustic Doppler Current Profiler) pour le courant et des marégraphes submersibles immergés pour la hauteur d'eau. La longueur moyenne de nos séries temporelles est 40 jours et sont réparties de 2007 à 2008.

Les résultats préliminaires sont concluants : bien que les composantes harmoniques aient été conformes à celles déjà établies par différents auteurs, les valeurs absolues des hauteurs d'eau ainsi que les phases présentent des écarts significatifs au moins dans deux zones. En outre, les hauteurs ont montré l'existence d'une boucle du courant général de la méditerrané qui pénètre dans le golfe de Gabès.

\section{Mots-clés :}

Génie côtier - Marée - Courant - Hydraulique maritime - Environnement littoral Composantes harmoniques - Circulation générale - Golfe de Gabès - Tunisie

\section{Introduction}

La dynamique du golfe de Gabès est étroitement liée à la circulation générale de la méditerranée d'une part, et d'autre part, aux propagations des ondes de marée à l'intérieur de ce golfe. Certes, les études de MOLINES (1991), ABDENNADHER \& BOUKTHIR (2006) et SAMMARI et al. (2006), se sont bien focalisés sur la dynamique du golfe de Gabés, contrairement à d'autres, qui ont axé leurs études sur la Méditerrané Orientale (tels que TSIMPLIS et al., 1995). Il est de plus en plus plausible, de confirmer ou d'infirmer leurs résultats par des mesures in situ, notamment dans la zone côtière.

\section{DOI: $10.5150 / \mathrm{cmcm} .2009 .027$}


Nous présentons dans la première partie les données de l'analyse harmonique. La deuxième portera sur les résultats de cette analyse et sur leurs comparaisons avec les études antérieures.

\section{Données}

Nous disposons, entre septembre 2007 et décembre 2008, de dix séries temporelles (hauteur d'eau obtenue par des marégraphes submersibles immergés et courants obtenus par des ADCP, voit tableau 1). Nous avons également exploité les séries obtenues par SAMMARI et al. (2006). Ces dernières enrichirons notre analyse et surtout rendront plus aisé la validation de nos mesures.

Tableau 1. Stations de mesures effectuées (durées en jours et profondeurs en mètres).

\begin{tabular}{lllllllll}
\hline Id & Instrument & Nom & Lon. & Lat. & $\Delta \boldsymbol{t}(\mathbf{s e c})$ & Début & Durée & Prof. \\
\hline 1 & RBR 2050 & Gabes 1 & $33^{\circ} 53^{\prime}$ & $10^{\circ} 07^{\prime}$ & 360 & $11 / 09 / 2007$ & 65.6 & 3.6 \\
2 & RBR 2050 & Taguermess & $33^{\circ} 49^{\prime}$ & $11^{\circ} 03^{\prime}$ & 360 & $12 / 09 / 2007$ & 64.5 & 3.2 \\
3 & RBR 2050 & Gabes 2 & $33^{\circ} 53^{\prime}$ & $10^{\circ} 07^{\prime}$ & 360 & $6 / 03 / 2008$ & 70.1 & 7.1 \\
4 & RBR 2050 & Cercina & $34^{\circ} 44^{\prime}$ & $11^{\circ} 05^{\prime}$ & 360 & $10 / 09 / 2007$ & 45.3 & 3.9 \\
5 & RBR 2050 & Mahres & $34^{\circ} 07^{\prime}$ & $10^{\circ} 25^{\prime}$ & 360 & $19 / 03 / 2009$ & 73.0 & 16 \\
6 & RBR 2050 & Elkantara & $33^{\circ} 39^{\prime}$ & $10^{\circ} 55^{\prime}$ & 360 & $28 / 05 / 2008$ & 40.9 & 3.2 \\
7 & Argonaut & Elkantara & $33^{\circ} 39^{\prime}$ & $10^{\circ} 55^{\prime}$ & 1200 & $28 / 05 / 2008$ & 40.9 & 3.2 \\
8 & ADCP & Taguermess & $33^{\circ} 49^{\prime}$ & $11^{\circ} 03^{\prime}$ & 1200 & $12 / 09 / 2007$ & 46.8 & 3.2 \\
9 & ADCP & Chebba & $35^{\circ} 06^{\prime}$ & $11^{\circ} 32^{\prime}$ & 3600 & $25 / 12 / 2008$ & 82.8 & 15 \\
10 & ADCP & Mahres & $34^{\circ} 07^{\prime}$ & $10^{\circ} 25^{\prime}$ & 3600 & $24 / 12 / 2008$ & 52.7 & 16 \\
\hline
\end{tabular}

\section{Méthodologies de travail. L'analyse harmonique (exploitation des mesures)}

C'est une méthode d'analyse de la marée permettant de la représenter sous forme d'une somme de composantes harmoniques de période bien déterminées, correspondant aux termes de la force génératrice astronomique luni-solaire et à des termes complémentaires d'origine hydrodynamique.

Cet outil est le fruit des travaux de GODIN (1972, 1976), leurs programmations sur ordinateur a été initié par FOREMAN (1977) sur Fortran puis repris par PAWLOWICZ et al. (2002) sur Matlab.

\section{Résultats et discussions}

\subsection{Amplitudes et phases}

En nous basant sur les résultats de l'analyse de la hauteur d'eau des différentes stations nous confirmons ceux de MOLINES (1991), TSIMPLIS et al. (1995) et ABDENNADHER \& BOUKTHIR (2006) pour la zone de Ganouch et la Skhira. En effet, il résulte bien que l'amplitude de $\mathrm{M}_{2}$ décroit en allant vers l'Est de $50 \mathrm{~cm}$ prés de Ganouch à $40 \mathrm{~cm}$ dans la partie Sud de Kerkennah. Les autres harmoniques principales 
selon l'ordre décroissant ci-après: $\mathrm{S}_{2}, \mathrm{~K}_{2}, \mathrm{~N}_{2}, \mathrm{~K}_{1}$ et $\mathrm{O}_{1}$ présentent les mêmes caractéristiques qualitatives, avec une amplitude réduite (de l'ordre de $30 \mathrm{~cm}$ pour $\mathrm{S}_{2}$ et $10 \mathrm{~cm}$ pour le reste).

Des écarts significatifs d'amplitude ont toutefois été observés autour de l'île de Djerba pouvant atteindre $22 \mathrm{~cm}$ pour $\mathrm{M}_{2}$ et $21 \mathrm{~cm}$ pour $\mathrm{S}_{2}$.

Nous estimons que ces écarts sont dus à une sous estimation des effets des petits fonds ( $<10 \mathrm{~m}$ qui sont loin d'être négligeables).

Un écart de phase des ondes de marée a été mis en évidence dans deux zones de ce golfe. Cet écart concerne les sous-bassins au niveau du canal de Kerkennah et de la lagune de Boughrara, (qui peut atteindre les $50^{\circ}$ ).

Les retards de pleine mer entre les différents sites ne sont pas seulement dus aux différences de longitude, mais également au frottement visqueux sur les petits fonds. Cette hypothèse est en cours de validation.

\subsection{Les courants}

Nous avons analysé les données de courants, qui ont montré que dans la station 9 (voir tableau 1) $47 \%$ de la variance est expliqué par la marée, et en allant vers l'intérieur du golfe (la station 10), 76\% est expliqué de la même manière. Alors qu'à Taguermess (station 8) la variance expliquée par la marée ne dépasse pas les 33\%. Le résiduel du courant à ces stations reste unidirectionnel et coïncide avec la ligne de côte moyenne. Nous en concluons que le courant général de la méditerrané comporte une ou plusieurs branches s'engouffrant dans le golfe de Gabès.

Pour la station 9 le flot est de direction Ouest-Nord-ouest pouvant atteindre la vitesse maximale de $15 \mathrm{~cm} \mathrm{~s}^{-1}$ et le jusant est dans la direction opposé avec un maximun légèrement superieur $\left(23 \mathrm{~cm} \mathrm{~s}^{-1}\right)$. Ces courant se composent avec un courant général Est-Sud-est de vitesse maximale égale à $38 \mathrm{~cm} \mathrm{~s}^{-1}$ (plus forte que celle des courant de marée) . A la station 10 (devant les îles Kneis), les courant de marée (flot: S-O, $24 \mathrm{~cm} \mathrm{~s}^{-1}$ et jusant : N-E, $30 \mathrm{~cm} \mathrm{~s}^{-1}$ ) doivent se composer avec un courant général N-E pouvant atteindre un maximun de vitesse de $30 \mathrm{~cm} \mathrm{~s}^{-1}$ avec une valeur moyenne de 15 $\mathrm{cm} \mathrm{s}^{-1}$. A la station 8 les courant de marée (flot: O-NO, $39 \mathrm{~cm} \mathrm{~s}^{-1}$ et jusant : E-SE, 65 $\mathrm{cm} \mathrm{s}^{-1}$ ) doivent se composer avec un courant général $\mathrm{O}-\mathrm{NO}$ pouvant atteindre un maximun de vitesse de $65 \mathrm{~cm} \mathrm{~s}^{-1}$ anec une valeur moyenne de $30 \mathrm{~cm} \mathrm{~s}^{-1}$.

Nous concluons, qu'il existe un contre courant au courant général de la méditerranné et qui lui est rattaché. Et d'ailleurs, il est de même allure que celui du golfe de Hammamet détecté par LCHF (1978).

Ce courant pourrait, sous toute probablité, pénétrer dans le golfe le long des côtes nord de l'île de Djerba et en ressortir au large de la poite sud des îles Kerkennah. 


\section{Conclusions}

Les mesures effectuées nous ont permis de calculer les composantes de la marée du golfe de Gabès, et ainsi vérifier qu'elles sont en cohérence avec les valeurs établies par les études antérieures. Elles ont, également, permis de préciser le chemin de propagation de cette onde de marée, notamment, aux alentours des îles de Kerkennah et Djerba. Accessoirement, nous avons constaté des écarts sensibles avec les valeurs calculées par ABDENNADHER \& BOUKTHIR (2006) et de repérer une branche du courant général de la Méditerrané qui se manifeste par un contre courant pénétrant dans le golfe de Gabès puis rejoignant le courant général qui longe les côtes sud du bassin méditerranéen.

\section{Références bibliographiques}

ABDENNADHER J., BOUKTHIR M. (2006). Numerical simulation of the barotropic tides in the Tunisian Shelf and the Strait of Sicily. Journal of Marine Systems $\mathrm{n}^{\circ} 63$, pp 162-182.

FOREMAN M.G.G. (1977). Manual for Tidal Heights Analysis and Prediction. Institute of Ocean Sciences, Patricia Bay, Victoria, B.C Pacific Marine Science Report $\mathrm{n}^{\circ} 77-10,97 \mathrm{p}$.

GODIN G.G. (1972). The Analysis of Tides. University of Toronto Press, 264 p.

LCHF -Laboratoire central d'hydraulique de France- (1978). Etude des ports de pêche côtière, études hydrauliques. Rapport n03A.

GODIN G.G. (1976). The reduction of current observations with the help of the admittance function. Marine Environmental Data Service Environment Canada. Ottawa, Technical Note $\mathrm{n}^{\circ} 14,13 \mathrm{p}$.

MOLINES J.M. (1991). Modeling the barotropic tides in the strait of Sicily and Tunisian coasts. Oceanol Acta $\mathrm{n}^{\circ} 14$ (3) pp 241-251.

PAWLOWICZ R., BEARDSLEY B., LENTZ S. (2002) Classical tidal harmonic analysis including error estimates in MATLAB using T_TIDE. Computer Geoscience, Pergamon Press, Inc. n²8, pp 929-937.

SAMMARI C., KOUTITONSKY V.G., MOUSSA M. (2006). Sea level variability and tidal resonance in the Gulf of Gabes, Tunisia. Continental Shelf Research $\mathrm{n}^{\circ} 26$, pp 338-350.

TSIMPLIS M., PROCTOR R., FLATHER R. (1995). A two dimensional tidal model for the Mediterranean Sea. Journal of Geophysical Research n 100. pp 16223-16239. 